\title{
Multi-omics analysis on seasonal variations of the biofilm microbial community in a full-scale pre- denitrification biofilter
}

Lu Tian

Ocean University of China - Laoshan Campus: Ocean University of China

Lin Wang ( $\square$ wangouc@163.com )

Ocean University of China - Laoshan Campus: Ocean University of China

Xiaofeng Zhang

qingdao water development service center

Xuda Huang

qingdao water development service center

Fuhao Wang

qingdao water affairs group environmental energy Co., Ltd

Sifu Zhu

qingdao haibo river water operation co., Itd

Xueqiang Li

qingdao haibo river water operation co., Itd.

Ying Guan

qingdao haibo river water operation co., Itd.

\section{Research Article}

Keywords: pre-denitrification biofilter, seasonal variations, microbial community composition, nitrogen metabolism, metabolic profiles

Posted Date: February 1st, 2022

DOl: https://doi.org/10.21203/rs.3.rs-1246390/v1

License: (c) (i) This work is licensed under a Creative Commons Attribution 4.0 International License. Read Full License 


\section{Abstract}

The seasonal variations of biofilm communities in a municipal wastewater treatment plant were investigated using multi-omics techniques. The abundance of the main phyla of microorganisms varied with season considerably, the Bacteroidetes enriched in winter and Chloroflexi in summer. The results of metaproteomic and metagenomic showed that most of the functional microorganisms belonged to the Betaproteobacteria class, and the abundance of Flavobacteria class enriched in winter make sure the denitrification performance to some extent. Seasonal variations affected the proteomic expression profiling, a total of 2835 differentially expressed proteins identified were significantly enriched in quorum sensing, two-component system, ribosome, benzoate degradation, butanoate metabolism, citrate cycle (TCA cycle), and cysteine and methionine metabolism pathways. With the expression of nitrogen metabolic proteins decreases in winter, the overall expression of denitrification-related enzymes in winter was much lower than that in summer, the nitrogen metabolism pathway varied significantly. Seasonal variations also induced the alteration of the biofilm metabolite profile; a total of 66 differential metabolites, 8 potential biomarkers, and 8 perturbed metabolic pathways such as TCA cycle were detected. It was found that most of the perturbed pathways are directly related to nitrogen metabolism, and several amino acids and organic acids associated with the TCA cycle were significantly perturbed, the accumulation of TCA cycle intermediates, ornithine and L-histidine in winter might be conducive to resisting cold temperatures. Furthermore, the correlation between biofilm microbial communities and metabolites was identified by the combined analysis of metabolomic and metaproteomic. The differences of microbial community structure, function and metabolism between winter and summer in a full-scale pre-denitrification biofilter were revealed for the first time, strengthening our understanding of the microbial ecology of biofilm communities.

\section{Introduction}

The principal objective of wastewater treatment plants (WWTPs) is to remove contaminants and nutrients from wastewater. As the most extensive application area of wastewater treatment, biological treatment can efficiently remove pollutants via its highly complex microbial consortium(Wang et al., 2012). Due to its high efficiency and low cost, activated sludge (AS) process and its variation processes, such as anaerobic/anoxic/aerobic (A2/0), anaerobic/oxic $(\mathrm{A} / \mathrm{O})$, and sequencing batch reactor (SBR), have become the mainstream processes in wastewater treatment plants all over the world (Chen et al., 2020; Liu et al., 2016; Zhang et al., 2016). Moreover, the A/O system is one of China's most commonly used AS processes (Jin et al., 2014). However, the superior advantages of the Biological Aerated Filter (BAF) system, such as higher hydraulic/organic loading rates, compact structure, and low sludge production over conventional AS, make it an attractive alternative (Pramanik et al., 2012; Tao et al., 2016).

The pre-denitrification biofilter system combines the A/O process and the BAF process, which has both advantages and practical application value. The denitrification biofilter is located at the front end of the system. The organic carbon in the influent is used as the electron donor for denitrification, and the nitrate in the effluent of the nitrification biofilter is recycled to the denitrification biofilter as the electron acceptor(Tian \& Wang, 2021). In contrast to activated sludge, massive amounts of microorganisms attach to the surface and pores of the filter media, forming biofilms (Zhou \& Xu, 2020). Biofilms are a highly complex microbial ecosystem composed of a variety of bacteria, viruses, eukaryotes and archaea (Tian \& Wang, 2021). Thus, the microbial biofilm is the prerequisite to ensure the well-performing of the pre-denitrification biofilter system. 
However, similar to WWTPs using AS process, the operation of the BAF system is also based on an empirical relationship between water quality indexes, operating parameters and reactor performance, and its reliability is not sufficient to maintain stable and efficient performance(Liu et al., 2016; Tian \& Wang, 2019; Werner et al., 2011). Microbial communities involved in WWTPs are affected by operational parameters, wastewater characteristics and seasonal variations (Latif et al., 2015) (Johnston et al., 2019). For WWTPs in temperate climate regions with distinct seasons, the microbial communities have apparent seasonality and temperature sensitivity (Johnston \& Behrens, 2020). Temperature is strongly correlated with microbial communities, which directly affects the performance of WWTPs (Tian \& Wang, 2020). Generally, the optimal temperature range for microorganisms is $20 \sim 35^{\circ} \mathrm{C}$, and the temperature in winter may drop below $10^{\circ} \mathrm{C}$ (Zhou \& Xu, 2019). It is still challenging to ensure that the treated effluent meets the regulatory standards at low temperatures, especially the WWTPs often experience seasonal nitrification failures in winter (Johnston et al., 2019). The study of seasonal variations is a possible way to link environmental factors with bacterial communities. Most studies on the microbial community dynamics affected by seasonal variations have focused on WWTPs with AS process, and the high-throughput sequencing method is generally employed (Johnston \& Behrens, 2020; Ju et al., 2014). However, the research on the BAF system is inadequate, and the information on the microbial gene composition has a limited role in elucidating gene expression and activity information. Therefore, the combined metagenomics, metaproteomics, and metabolomics methods were used to investigate the seasonal variations of the biofilm microbial community composition, functions, and metabolic profiles of a full-scale municipal WWTP with a pre-denitrification biofilter system. The WWTP is located in a temperate maritime monsoon climate zone, the temperature difference between winter and summer makes us possible to compare the samples' community dynamics and performance parameters by season. Our data present a systematic understanding of the seasonal variations of the biofilm microbial communities of full-scale biofilm WWTP, which can further provide meaningful guidance for manipulating microbial communities in a manner that improves treatment efficiency and stability.

\section{Materials And Method}

\subsection{Wastewater treatment plant and sample collection}

The samples collected in this study were from the Maidao WWTP, Qingdao, China. The biological treatment process of the WWTP is a pre-denitrification biofilter system, consisting of 14 anaerobic BAFs, 7 aerobic BAFs and 7 BIOSTYR BAFs. The specific influent characteristics and process parameters are as described previously.

Biofilm samples were taken from the No.7 anaerobic BAF in January and July 2019, respectively. And the BAF operated steadily and effectively during the sampling period. The operational conditions of sampling were summarized in Table 1. To sure the uniformity and representativeness of the sampling, samples were collected three times a month at different heights of the material layer in the filter and then mixed them. 
Table 1

Operational conditions and nitrogen removal performance of the pre-denitrification biofilter

\begin{tabular}{|c|c|c|c|c|c|c|c|c|c|}
\hline Date & $\begin{array}{l}\text { OD } \\
(\mathrm{mg} / \mathrm{L})\end{array}$ & $\begin{array}{l}\text { OPR } \\
(\mathrm{mV})\end{array}$ & $\begin{array}{l}\text { T } \\
\text { (Water, } \\
\left.{ }^{\circ} \mathrm{C}\right)\end{array}$ & $\begin{array}{l}\text { Reflux } \\
\text { Ratio }\end{array}$ & $\begin{array}{l}\text { Hydraulic } \\
\text { Load } \\
\left(\mathrm{m}^{3} /\left(\mathrm{m}^{3}\right.\right. \\
\text { filter } \\
\text { material } \cdot h))\end{array}$ & $\begin{array}{l}\mathrm{TN}_{\mathrm{lnf}} \\
(\mathrm{mg} / \mathrm{L})\end{array}$ & $\begin{array}{l}\mathrm{TN}_{\text {eff }} \\
(\mathrm{mg} / \mathrm{L})\end{array}$ & $\begin{array}{l}\mathrm{NH}_{4}{ }^{+}{ }_{\mathrm{Inf}} \\
(\mathrm{mg} / \mathrm{L})\end{array}$ & $\begin{array}{l}\mathrm{NH}_{4}{ }^{+} \mathrm{Ef} \\
(\mathrm{mg} / \mathrm{L})\end{array}$ \\
\hline $2019 / 1 / 3$ & 0.34 & -143.73 & 15.7 & 2.67 & 4.82 & 56.15 & 15.00 & 46.80 & 3.59 \\
\hline 2019/1/10 & 0.39 & -104.37 & 14.4 & 2.51 & 3.75 & 51.50 & 14.99 & 46.60 & 0.14 \\
\hline 2019/1/17 & 0.30 & -165.90 & 14.2 & 2.00 & 3.94 & 50.60 & 14.91 & 45.80 & 0.08 \\
\hline 2019/7/1 & 0.19 & -236.00 & 22.0 & 3.08 & 4.76 & 44.53 & 10.16 & 32.50 & 0.11 \\
\hline 2019/7/17 & 0.15 & -255.00 & 23.1 & 3.04 & 4.88 & 42.48 & 12.38 & 32.75 & 0.31 \\
\hline $2019 / 7 / 31$ & 0.17 & -248.00 & 21.9 & 3.15 & 4.72 & 36.30 & 11.03 & 29.60 & 0.26 \\
\hline
\end{tabular}

\subsection{Metagenomic analysis}

\subsubsection{DNA extraction and sequencing}

Genomic DNA was extracted from the samples using the E.Z.N.A.® Soil DNA Kit (Omega Bio-tek, USA) and detected by $1 \%$ agarose gel electrophoresis. DNA fragmentation ( 400bp) was done with Covaris M220 Focused-ultrasonicator (Covaris, USA) and followed by Paired-end library construction using the NEXTFLEX ${ }^{\mathrm{TM}}$ Rapid DNA-Seq Kit (Bioo Scientific, USA). Finally, the prepared libraries were subjected to Illumina HiSeq 4000 platform (Majorbio Bio-Pharm Co., Ltd, Shanghai, China) for metagenomic analysis.

\subsubsection{Taxonomic assignment and functional annotation}

To ensure the accuracy of the subsequent analysis results, we processed the raw reads using fastp (https://github.com/OpenGene/fastp) for quality control to obtain clean reads. MEGAHIT version 1.1.2 was used for gene assembly, and MetaGene (http://metagene.cb.k.u-tokyo.ac.jp/) was used for gene prediction. First, the predicted gene sequences of all samples were clustered using CD-HIT (http://www.bioinformatics.org/cd-hit/) to construct a non-redundant gene set. Next, the clean reads of each sample were compared with the non-redundant gene set separately by SOAPaligner version 2.21 to count the abundance information of genes in the corresponding samples. Subsequently, the Non-redundant gene set was compared against the NR database (ftp://ftp.ncbi.nlm.nih.gov/blast/db/) for taxonomic composition and abundance information using BLASTP version 2.3.0 (ftp://ftp.ncbi.nlm.nih.gov/blast/executables/blast+/2.3.0/, e-value = 1e-5). Finally, the nonredundant gene set sequences were aligned with the KEGG database (http://www.genome.jp/kegg/). Based on the alignment results, the functional annotation was performed using KOBAS 2.0.

\subsection{Metaproteomic analysis}

\subsubsection{Protein extraction, trypsin digestion, and LC-MS/MS analysis}


Three replicates were obtained from summer and winter samples on January 3, 10, 17 and July 2, 3, 5, respectively. The Borax/PVPP/Phenol (BPP) method based on a published protocol was used to extract protein. And the Bicinchoninic Acid (BCA) method was used to determine the protein concentration. Trypsin digestion and LC-MS/MS analysis were then performed, with the protocol described in our previous study (Tian \& Wang, 2021).

\subsubsection{Protein database, functional annotation, and differential analysis}

Peak identification was first carried out for the raw files of the LC-MS/MS analysis. And then, a reference database was created, including all proteins from the summer and winter metagenomic assemblies for peptide and protein identification. All identified proteins were subject to taxonomic assignment, functional classification annotation and pathway annotation using NR database, GO database (http://www.geneontology.org) and KEGG database. Student's t-test was performed to screen differential proteins according to the fold change (FC $<0.50$ or $\mathrm{FC}>2.00)$ and significant $P$ values $(\mathrm{p}<0.05)$.

\subsection{Metabolomic analysis}

Six parallel samples were set up for each sample to avoid experimental errors. Sample preparation, derivatization, chromatographic and mass spectrometric conditions followed the previously described untargeted GC-MS metabolomics protocol (Tian \& Wang, 2021). The raw data was pre-processed using MassHunter workstation Quantitative Analysis (v10.0.707.0) for low-quality peak filtering, missing value recording, data normalization, and logarithmic transformation. The pre-processed data was submitted to the Majorbio Cloud Platform (www.majorbio.com) for principal component analysis (PCA), orthogonal least squares discriminant analysis (OPLS-DA) and student's t-test analysis. Variable importance in projection (VIP) value based on the OPLS-DA model and $p$-value based on student's t-test were used to identify the differential metabolites (VIP $>1$ and $p<$ 0.05).

\section{Results And Discussion}

\subsection{Seasonal variations in the biofilm microbiome}

The response of the biofilm microbiome to seasonal variations in a denitrification biofilter of the Maidao wastewater treatment plant was investigated. DNA was extracted from summer and winter biofilm samples, respectively, and the two metagenomes were sequenced using Illumina HiSeq. A total of 9.44 million raw pairedend reads were generated with a length of $150 \mathrm{bps}, 9.33$ million high-quality clean reads were retained after quality control. 1,064,671 contigs for gene prediction were obtained using the multiple hybrid assembly strategy, and 1,644,199 gene sequences were yielded (Table S1). Bacteria were overwhelmingly dominant among biofilm microorganisms, with over $99.5 \%$ of the gene sequences annotated as bacteria and $0.05 \%$ of the residual as viruses, eukaryote, and archaea.

For the Bacteria domain, a total of 79 phyla were detected in all samples. As shown in Fig. 1a, the top 5 phyla were Proteobacteria (69.4\%), Bacteroidetes (24.5\%), Actinobacteria (0.9\%), Chloroflexi (0.7\%) and Firmicutes (0.6\%). The bacteria phyla mentioned above are commonly found in municipal WWTPs worldwide with different biological treatment processes, and Proteobacteria and Bacteroidetes account for the largest proportion of microorganisms (de Celis et al., 2020; Wang et al., 2016). Proteobacteria is a highly diverse phylum that involved 
in various anaerobic reactions (Greses et al., 2018). A study of nirS-Type Denitrifying Bacterial Community from 18 WWTPs found that Proteobacteria was dominated in all samples (Zhang et al., 2019). Bacteroidetes phylum has a strong metabolic capacity for proteins, lipids and other complex macromolecular organic matter. Chloroflexi is involved in the anaerobic degradation of glucose and complex organic matter (e.g., cellulose) and plays a role in nitrite reduction (Hug et al., 2013).

Although the major phyla were the same in samples from different seasons, their respective abundances were quite different. It is noteworthy that the percentage of Bacteroidetes phylum was $28.3 \%$ in the summer sample, $71.7 \%$ in the winter sample (Table S2). In contrast, Chloroflexi phylum was accounted for $90.7 \%$ in the summer sample and $9.3 \%$ in the winter sample. The higher relative abundance of Bacteroidetes in winter samples might be explained by some subdivisions of Bacteroidetes affiliated with psychrophilic and psychrotolerant organisms, which are enriched in cold environments and develop mechanisms of cold resistance (Ducey et al., 2010). Within Chloroflexi, Anaerolineae was the predominant class, followed by Chloroflexia. The relative abundance of Anaerolineae was reported to be decreased with temperature decrease (Damodara Kannan et al., 2020). Moreover, Chloroflexia's representative nutritional mode is photosynthesis (Ward et al., 2018), which may grow on the external layer of the biofilm and is thus strongly influenced by temperature, consistent with the previously reported greater abundance of green algae with increasing temperature (Uribe-Lorio et al., 2019). The abundance of Proteobacteria, Actinobacteria and Firmicutes varied little in the winter and summer samples, showed strong stability to seasonal changes. Previous studies on activated sludge found that the abundance of Proteobacteria in activated sludge samples was much higher in winter than in other seasons, while the abundance of Bacteroidetes was lower than in other seasons (Ju et al., 2014; Liu et al., 2016). A possible explanation for the different results is the structural difference between biofilm and activated sludge.

Protein sequences from metaproteomic were compared with NR database to obtain taxonomic annotations and abundance information. Subsequently, taxonomic annotation results of metagenomics and metaproteomic were compared at the class level for different season samples. Betaproteobacteria class and Alphaproteobacteria class were dominant bacteria (> 55\%) in the classification results of both seasons and both annotation methods. For both season samples, Betaproteobacteria were much more abundant in the metaproteomic classification than in the metagenomic, while the abundance of Alphaproteobacteria was lower than that in the metagenomic classification (Fig. 1b \& c). Therefore, the majority of functional microorganisms in denitrification biofilter belonging to Betaproteobacteria. Betaproteobacteria are the majority of denitrifying bacteria found in denitrification processes (Wang \& Chu, 2016). It is noteworthy that Flavobacteriia, an essential member of biofilm microorganisms, was significantly abundant in the winter sample, in agreement with a previous study that Flavobacteriia increased in abundance under cold water conditions (Kraemer et al., 2020), the enhanced abundance of Flavobacteriia in winter ensured the removal performance of nitrogen (Zhou et al., 2020).

\subsection{Proteomic expression profiling and differentially expressed proteins identification}

The label-free protein expression quantification method was used to evaluate the effect of seasonal variations on denitrification biofilter microorganisms. The LC-MS/MS analysis generated 99,307 Peptide-Spectrum Matches, 19,309 peptides and 27,305 unique peptides in the database of 375,757 spectra, and a total of 7,767 proteins were identified (Fig. S1a). The gene ontology (GO) analysis grouped all proteins into three major categories: biological process (BP), cellular component (CC), and molecular function (MF) (Fig. S1b). The catalytic activity 
and binding were the dominant categories in MF ontology. Among the BP ontology, the majority of proteins were attributed to cellular processes and metabolic processes. And cellular anatomical entity in cellular component had large numbers of proteins. Based on the annotation result of KEGG analysis, most proteins were primarily involved in global and overview maps, energy metabolism and carbohydrate metabolism pathways belonging to the metabolism category (Fig. S1C). The Principal Component Analysis (PCA) score plot exhibited that the biofilm samples from summer and winter were significantly separated, indicating that the proteomic expression profiling of the biofilm shifted with the seasons (Fig. 2a).

To further access the dynamic changes of proteins in biofilms during summer and winter, 2835 differentially expressed proteins (DEPs) were identified from the two groups of samples using a Screening Criteria of fold change $\geq 2.00$ or $\leq 0.50$ and $P<0.05$. There were 1596 up-regulated and 1239 down-regulated DEPs in summer samples compared with the winter samples (Fig. 2b). The top 15 DEPs ranked according to P-value were listed in Table S3, which may play an important role in seasonal adaptation and may be further investigated as candidate genes in the future.

GO enrichment analysis based on DEPs was conducted to clarify the functional differences of samples between seasons (Fig. 2c). The results showed that in response to seasonal variations, GO terms belonging to BP and MF ontologies had high enrichment factors with strong significance $(P<0.05)$. In BP ontology, cellular process, ATP metabolic process, small molecule metabolic process, organic substance metabolic process and biosynthetic process were significantly enriched. In MF ontology, most of the DEPs were enriched in carbohydrate derivative binding, small molecule binding, heterocyclic compound binding and organic cyclic compound binding. Detailed information about GO enrichment analysis is shown in Table S4. In addition, the most enriched pathways related to the binding of ribonucleotide, nucleotide, and nucleoside phosphate. Nucleotide-binding proteins are essential protein families involved in various critical cellular processes such as cell signaling (Xiao \& Wang, 2016). In addition, several GO terms related to transporter activity and biosynthetic and metabolic processes of organic acids were also statistically significantly enriched.

KEGG pathway enrichment analysis was further conducted based on DEPs. DEPs were assigned to $247 \mathrm{KEGG}$ pathways, with most of them (116) related to metabolism (M), 48 pathways to human disease (HD), 28 to organismal systems (OS), 24 cellular processes (CP) pathways, 17 pathways for environmental information processing (EIP), and 14 pathways for genetic information processing (GIP). Among the enriched KEGG pathways (Fig. 2d), seven pathways, including quorum sensing, two-component system, ribosome, benzoate degradation, butanoate metabolism, citrate cycle (TCA cycle), and cysteine and methionine metabolism were significantly enriched $(p<0.01)$. Except for the two-component system, the other six pathways are mainly enriched by upregulated DEPs. The ribosome and cysteine and methionine metabolism had the highest significant enrichment levels $(p=0.0007)$. Quorum sensing plays a vital role in arranging bacterial gene regulation, participating in the regulation of biofilm formation and extracellular polymer production processes, enabling bacteria to adapt to changes in environmental conditions (Acet et al., 2021). Temperature is a significant factor affecting Quorum sensing (Wang et al., 2019), and a study of anaerobic ammonium oxidation biofilm reactor found that a decrease in temperature weakened quorum sensing and deteriorated nitrogen removal performance (Liu et al., 2021). The enrichment of the quorum sensing pathway implies its role in the adaptation of biofilm microbiome to seasonal variations. The two-component system is a fundamental stimulus-response coupling mechanism that enables microorganisms to sense and respond to changes in environmental conditions (Kuang et al., 2018). Following seasonal variations, some proteins involved in the two-component system pathway were differentially regulated. 
There was significant regulation of proteins involved in the degradation/metabolism of organic acids (such as Benzoate, Butanoate) and amino acids (Cysteine and methionine metabolism). Benzoate conversion into TCA cycle intermediates via benzoyl-CoA (Garrido-Sanz et al., 2018). Butanoate metabolism is directly linked to the TCA cycle via succinate and indirectly linked via pyruvate and acetyl-CoA (Goudarzi et al., 2015). Similarly, GO terms associated with the organic acid metabolic process were significantly enriched. In bacteria, methionine is synthesized from aspartate and cysteine is metabolized to pyruvate through various pathways (Park \& Lee, 2010; Samuilov et al., 2018), whereas aspartate and pyruvate are important compounds in the TCA cycle. All these pathways are directly or indirectly linked to the TCA cycle, through which their degradation products generate the reducing agent $\mathrm{NADH}$ to be used as an electron donor for denitrification (Emmanuel et al., 2019a). The nitrogen metabolic pathway was also one of the significant pathways with 181 up-regulated and 61 down-regulated DEPs, which validates the enhanced efficiency of denitrification in summer. And the most significant pathway, ribosomes, was also significantly enriched in the up-regulated DEP ( $p=0.007$, Fig. S2), demonstrating the extensive enhancement of protein expression and synthesis in summer to meet the greater demands of cell growth (Zhong et al., 2019).

\subsection{DEPs involved in the nitrogen metabolism}

Based on the metagenomic results, the nitrogen metabolism pathway was the most active in the predenitrification biofilter, with the denitrification module and DNRA module, the most represented functions; and there were significant differences in denitrification, nitrate/nitrite transport systems, and nitrate assimilation modules (Fig. S3a, b). According to the results of metaproteomics, the expression of proteins for nitrogen metabolism was lower than carbon metabolism and Glyoxylate and dicarboxylate metabolism in summer. In contrast, the expression of proteins for nitrogen metabolism decreased significantly in winter, the sixth in the ranking of the abundance of all pathways (Fig. S3c). The above metagenomic and metaproteomic results showed that the nitrogen metabolism pathway differs significantly between summer and winter.

To further investigate the variation of nitrogen metabolism in different seasons, key genes involved in the nitrogen metabolism pathway were identified by KEGG analysis of DEPs. The KEGG pathway map (Fig. 3a) showed that the nitrogen metabolism pathway was enriched mainly by up-regulated proteins. EC 1.7.5.1 is known as nitrate reductase (alpha subunit, beta subunit and gamma subunit, NarGHI), and EC 1.7.99.4 is the periplasmic nitrate reductase (NapA), both responsible for the conversion of nitrate to nitrite. Nitrite is reduced to nitric oxide by nitrite reductase (NirKS), denoted as EC 1.7.2.1 in the KEGG pathway map. EC 1.7.2.5 marks the conversion of nitric oxide to nitrous oxide by nitric oxide reductase subunit B (NorB). The last step of denitrification is catalyzed by nitrous-oxide reductase (NosZ), denoted as EC 1.7.2.4. The key enzymes mentioned above were commonly expressed in both seasons, with the abundance of up-regulated proteins was much higher than that of downregulated proteins, and the expression of denitrification related enzymes in summer was at least as twice high as in winter samples. It could explain the decreased denitrification performance of the pre-denitrification biofilter system in winter (Table 1). In addition, nitrate transporter (Nrt) was entirely enriched by up-regulated proteins to uptake extracellular nitrate. The ammonia input-related enzymes such as carbonic anhydrase (Can) and glutamate dehydrogenase (Gdh) were enriched by up-regulated proteins. Glutamine synthetase (GIn) was highly enriched by down-regulated proteins and was expressed more vigorously in winter, and glutamate synthase (Glu) was slightly more expressed in summer than in winter.

Four potential metabolic reactions in nitrogen metabolism were identified, including denitrification, dissimilatory nitrate reduction, assimilatory nitrate reductase and nitrification (Fig. 3b). Genes encoding the alpha and beta 
subunits of nitrate reductase (narG and narH) were generally actively expressed in summer samples. However, the genes LPB072_22010, Tmz1t_2635 and Tchl_2559 encoded the alpha subunit of nitrate reductase, AzCIB_2182 and LPB072_22005 encoding the beta subunit, as well as the gene Dtpsy_0430 encoding gamma subunit, were significantly better expressed in winter samples. These bacteria belong to the family Comamonadaceae and Zoogloeaceae of the order Betaproteobacteria, core microorganisms in low-temperature bioreactors (GonzalezMartinez et al., 2018; Huang et al., 2014), and can therefore accumulate at low temperatures in winter to improve the performance of denitrification. The genes encoding narG and narH can also encode nitrite oxidoreductase. However, under the premise of anaerobic conditions in the denitrification filter, nitrite oxidoreductase cannot be expressed and nitrification reaction does not occur (Kampschreur et al., 2009). In the DNRA pathway, the first step of nitrate reduction is consistent with the denitrification pathway. The presence of genes encoding nirBD and nrfAH was detected by metagenomics (Tian \& Wang, 2021), but the expression of related proteins was not detected, thus DNRA pathway excluded in this study. Nitrite reductase was the most actively expressed enzyme in this study, with NirS accounting for $56 \%$ of the DEPs involved in nitrogen metabolism. The species composition of the denitrifying bacterial community encoding NirS in summer was significantly different from that in winter. The most abundant bacteria, such as Thauera chlorobenzoica (Tchl_2591), Dechloromonas aromatica (Daro_3323) and Acidovorax sp. JS42 (Ajs_1912), all encoded and expressed genes for nitrite reductase (NirS) and were significantly up regulated in summer. The expression of nirS-encoding genes was much higher in summer than that in winter, and the expression of nirk-encoding genes showed the opposite pattern. Genes encoding Nirk showed low expression and was significantly downregulated $(p<0.01)$ in summer samples, suggesting that the NirK may play an important role in nitrite reduction in winter. The Competition between denitrifying bacteria encoding NirS and NirK could be responsible for the low expression of genes encoding NirK in summer (Shi et al., 2019). Based on the expression of genes encoding nitric oxide reductase, nitrous oxide in denitrification biofilters may be produced mainly by Elizabethkingia, Rubrivivax and Poseidonibacter in winter. Notably, a substantial proportion of the genes encoding NosZ were not significantly differentially expressed in the samples of the two seasons, whose protein expression was 1.7-fold higher and $~ 3.0$-fold higher than that of the up-regulated and the down-regulated protein. It is consistent with a previous study in which season had less influence on the nosZ community (Ross et al., 2020). Certain bacteria, such as Capnocytophaga (chg:

AXF12_04010), Polaromonas (pna: Pnap_3762) and Hydrogenophaga (hyr: BSY239_3898) showed the capability to express assimilatory nitrate reductase in this study. In addition, Polaromonas and Hydrogenophaga, both belonging to Comamonadaceae class, can also produce Nrt to transport extracellular nitrate and nitrite to the intracellular compartment. Even though metagenomics detected multiple nitrogen metabolic pathways, the metaproteomic results validated only two pathways: assimilative nitrate reduction and denitrification. And the genes for denitrification showed the highest protein expression.

\subsection{Metabolomic analysis}

Microbial cells can adapt to certain shifts in environmental conditions by exhibiting corresponding responses through changes in relevant metabolic processes (Dai et al., 2017). It is crucial to reveal community metabolic profiling and identify the relationship between metabolic activities and seasonal variations. By resolving the endproducts and intermediates of cellular metabolism, metabolomics is supposed to be the most sensitive indicator of the phenotype of the whole community and allows to derive critical metabolic processes (Roume et al., 2015). Therefore, to complement the results of metagenomic and metaproteomic analyses, metabolomic analysis was performed to investigate the changes in metabolites of the two groups of biofilm samples. 


\subsubsection{Biofilm metabolite profile and impact of season variations on community metabolome}

Using GC-MS, a total of 248 metabolites were identified in biofilms. PCA was performed among the QC samples and tested samples. The score plot (Fig. 4a) revealed a clear separation of biofilm samples from the winter and summer groups along with the first principal component (PC1), explaining $35.4 \%$ of the variance within the data. The QC samples were well grouped, tightly clustered with each other and separated from the tested samples, which confirmed the good analytical performance and data quality of GC-MS and supported that the separation between groups was related to biological variations (Cala et al., 2018). The corresponding loading plot (Fig. 4b) reflected the distribution of metabolites in the biofilm samples, with metabolites further away from the center (such as Lanosterol, Glycerol 3-phosphate and Phosphogluconic Acid) contributing more to the distinction of the samples. Further multivariate analysis using supervised OPLS-DA model was performed to predict variables of interest to discriminate the summer group from the winter group (fa), and the two principal components explained $49.3 \%$ of the variance. The corresponding stochastic model of R2 and Q2 values showed that the intercept of the Q2 was less than 0 and the model was not overfitted (Fig. S4b). These results indicated that seasonal variations induced alteration in the biofilm metabolite profile.

The student's t-test (Fig. S4c) showed that a total of 80 metabolites were significantly different $(p<0.05)$ between the two groups, together with the screening criterion of OPLS-DA model VIP $>1$, a total of 66 differential metabolites were identified. All differential metabolites were assigned to the KEGG database, and 28 of which were matched and classified: Peptides (8), Organic acids (6), Carbohydrates (4), Hormones and transmitters (3), Lipids (1), Nucleic acids (1), Steroids (6) and Vitamins and Cofactors (2) (Fig. S4d). Pathway topology analysis was performed to reveal the perturbed metabolic pathways based on the identified differential metabolites and changes in their concentrations. Eight pathways with pathway impact values above 0.1 were selected as perturbed metabolic pathways (Fig. 4d, Table S5), including pyruvate metabolism, butanoate metabolism, glycerolipid metabolism, glycerophospholipid metabolism, pantothenate and CoA biosynthesis and alanine, aspartate and glutamate metabolism, valine, leucine and isoleucine biosynthesis and citrate cycle (TCA cycle). Further enrichment analysis (Fig. 4e) confirmed that pyruvate metabolism, butanoate metabolism, pantothenate and CoA biosynthesis, alanine, aspartate and glutamate metabolism and TCA cycle were significantly affected ( $p$ $<0.05$ ) by seasonal variations. Fold change thresholds were included based on the selected differential metabolites, three metabolites were up-regulated (foldchange $>1.5$ ) in the summer group, and five metabolites were down-regulated (foldchange $<0.67$ ). These eight metabolites were significantly regulated and selected as potential biomarkers (Fig. 4c). The metabolic profiling association between potential biomarkers and perturbed metabolites was further analyzed (Fig. 5b). Changes in potential biomarkers were found to be correlated with changes in most metabolites involved in the perturbation pathway $(|r|>0.4, p<0.05)$, suggesting that changes in biomarkers can reflect reprogramming of metabolic pathways during seasonal changes.

Notably, most perturbed pathways were directly related to nitrogen metabolism, such as the TCA cycle and the metabolism of pyruvate, butanoate, alanine, aspartate, and glutamate. Several amino acids and organic acids related to TCA cycle were significantly perturbed by seasonal variation (Fig. 5a). Amino acids are essential to bacteria and components of all proteins, playing a critical role in microbial physiology processes, such as forming bacterial cell walls, synthesizing key enzymes, and being utilized as carbon source and nitrogen source (Aliashkevich et al., 2018; Cava et al., 2011). Aspartate, L-Aspartic acid, L-Tyrosine and L-Phenylalanine were up- 
regulated, while ornithine and L-Histidine were down-regulated. In addition to the regulation of amino acids, five organic acids (TCA cycle intermediates), including pyruvic acid, isocitric acid, butanedioic acid, fumaric acid and malic acid were significantly elevated in winter. The denitrification pathway could be associated with the degradation of amino acids and organic acids, with their degradation products going through the TCA cycle and producing NADH used as electron donor for denitrification (Emmanuel et al., 2019b). The biosynthesis and metabolism of amino acids and organic acids are closely linked through the TCA cycle (Zhao et al., 2019), therefore the metabolite alteration caused by seasonal variation affect both carbon metabolism and nitrogen metabolism. Moreover, many amino acids can act as compatible solutes due to their kosmotropicity, which allows them to stabilize membranes and proteins against environmental stresses (He et al., 2016; Xu et al., 2020). The accumulation of TCA cycle intermediates, ornithine and L-Histidine in winter may be a defense or coping strategy against the cold temperature (He et al., 2016).

\subsubsection{Correlation study of biofilm microbiome and community metabolites}

An integrated analysis of metabolomic and metaproteomic was performed for the two groups to explore and define the relationship between microbial communities and metabolic functions. Correlation analysis of the association between microbial communities with differential metabolites provided insight into the composition and function of microbial communities (Fig. 5c). Bacteroidetes with higher abundance in winter was negatively associated $(p<0.05)$ with up-regulated metabolites such as L-Aspartic acid, D-Fructose and mevalonic acid. While Chloroflexi with higher abundance in summer was negatively associated with down-regulated metabolites such as ornithine, L-Histidine and isocitric acid, suggesting that alterations in metabolite profiling of microbial communities reflect shifts in microbial community dynamics. Among the potential biomarkers, nicotinamide was positively associated with Streptophyta and Chlorophyta belonging to Viridiplantae, as nicotinamide is a metabolite synthesized by all plants and can react with 2-oxoglutamic acid to form L-glutamic acid, which is the largest contributor to the intermediate fluxes of the TCA cycle (Ohata et al., 1993; Takahashi et al., 1999). In addition, nicotinamide was also positively associated with fungi such as Ascomycota and Mucoromycota, and its presence in certain fungi was confirmed by previous studies (Laffont \& Arnoux, 2020). Only trace amounts of lanosterol were detected in summer samples, while it was significantly elevated in winter. Lanosterol was significantly associated with Chordata and Rotifera, which belong to metazoan, and it is the initial biosynthetic sterol in the metazoa sterol pathway (Kodner et al., 2008). Other potential biomarkers were also positively or negatively associated with eukaryotes, such as fungi, metazoa and viridiplanta, as eukaryotes may respond to seasonal changes with different resistance and sensitivity (Zhang et al., 2020). Our study revealed that seasonal variations directly impact microbial communities and metabolites and that there is a correlation between them. However, the relationship between metabolites and microbial communities in this study was purely correlational without further controlled experiments, and the control experiment should be conducted to find a detailed relation in the future.

\section{Conclusion}

This is the first integrative study that applied metagenomic, metaproteomic and metabolomic analyses to investigate structural, functional and metabolic variations in the microbial community of a pre-denitrification biofilter during the winter and summer months. Season variations were shown to directly influence the microbial community composition, functional traits, key enzymes, and critical metabolic pathways in the biofilm. The

Page $11 / 23$ 
perturbed pathways were directly related to nitrogen metabolism, and genes involved in nitrogen metabolism and key denitrifying enzymes were decreased in winter, responsible for the weakened denitrification performance. Our results demonstrate that multi-omics can be a powerful tool for identifying biofilm microbial responses to seasonal variations and that this approach can be transferred to other studies of adverse external environmental effects, which allows us to strengthen insights into wastewater biofilm ecology and optimize reactor operation.

\section{Declarations}

\section{Ethics approval}

Not applicable.

\section{Consent to participate}

Not applicable.

\section{Consent for publication}

Not applicable.

\section{Author Contributions}

All authors contributed to the study conception and design. Formal analysis, Investigation and Visualization were performed by Lu Tian and Lin Wang. Software and Data Curation were performed by Xiaofeng Zhang, Xuda Huang and Fuhao Wang. Resources and Supervision were performed by Sifu Zhu,Xueqiang Li and Ying Guan. The first draft of the manuscript was written by Lu Tian and all authors commented on previous versions of the manuscript. All authors read and approved the final manuscript.

\section{Funding}

This work was supported by Science and Technology Program for Public Wellbeing of Qingdao [21-1-4-sf-11-nsh].

\section{Competing interest}

The authors declare that they have no known competing financial interests or personal relationships that could have appeared to influence the work reported in this paper.

\section{Availability of data and materials}

The datasets used and/or analyzed during the current study are available from the corresponding author on reasonable request.

\section{References}

1. Acet, O., Erdonmez, D., Acet, B.O., Odabasi, M. 2021. N-acyl homoserine lactone molecules assisted quorum sensing: effects consequences and monitoring of bacteria talking in real life. Arch Microbiol. https://doi.org/10.1007/s00203-021-02381-9 
2. Aliashkevich, A., Alvarez, L., Cava, F. 2018. New Insights Into the Mechanisms and Biological Roles of DAmino Acids in Complex Eco-Systems. Frontiers in Microbiology, 9, 11.

https://doi.org/10.3389/fmicb.2018.00683

3. Cala, M.P., Agullo-Ortuno, M.T., Prieto-Garcia, E., Gonzalez-Riano, C., Parrilla-Rubio, L., Barbas, C., Diaz-Garcia, C.V., Garcia, A., Pernaut, C., Adeva, J., Riesco, M.C., Ruperez, F.J., Lopez-Martin, J.A. 2018. Multiplatform plasma fingerprinting in cancer cachexia: a pilot observational and translational study. J Cachexia Sarcopenia Muscle, 9(2), 348-357. https://doi.org/10.1002/jcsm.12270

4. Cava, F., Lam, H., de Pedro, M.A., Waldor, M.K. 2011. Emerging knowledge of regulatory roles of D-amino acids in bacteria. Cell Mol Life Sci, 68(5), 817-31. https://doi.org/10.1007/s00018-010-0571-8

5. Chen, H., Wang, M., Chang, S. 2020. Disentangling Community Structure of Ecological System in Activated Sludge: Core Communities, Functionality, and Functional Redundancy. Microb Ecol, 80(2), 296-308. https://doi.org/10.1007/s00248-020-01492-y

6. Dai, H., Dai, Z., Peng, L., Wu, Y., Zou, H., Lu, X. 2017. Metagenomic and metabolomic analysis reveals the effects of chemical phosphorus recovery on biological nutrient removal system. Chemical Engineering Journal, 328, 1087-1097. https://doi.org/10.1016/j.cej.2017.07.119

7. Damodara Kannan, A., Evans, P., Parameswaran, P. 2020. Long-term microbial community dynamics in a pilot-scale gas sparged anaerobic membrane bioreactor treating municipal wastewater under seasonal variations. Bioresour Technol, 310, 123425. https://doi.org/10.1016/j.biortech.2020.123425

8. de Celis, M., Belda, I., Ortiz-Alvarez, R., Arregui, L., Marquina, D., Serrano, S., Santos, A. 2020. Tuning up microbiome analysis to monitor WWTPs' biological reactors functioning. Sci Rep, 10(1), 4079. https://doi.org/10.1038/s41598-020-61092-1

9. Ducey, T.F., Vanotti, M.B., Shriner, A.D., Szogi, A.A., Ellison, A.Q. 2010. Characterization of a microbial community capable of nitrification at cold temperature. Bioresour Technol, 101(2), 491-500. https://doi.org/10.1016/j.biortech.2009.07.091

10. Emmanuel, S.A., Sul, W.J., Seong, H.J., Rhee, C., Ekpheghere, K.I., Kim, I.S., Kim, H.G., Koh, S.C. 2019 a. Metagenomic analysis of relationships between the denitrification process and carbon metabolism in a bioaugmented full-scale tannery wastewater treatment plant. World J Microbiol Biotechnol, 35(10), 149. https://doi.org/10.1007/s11274-019-2716-8

11. Emmanuel, S.A., Sul, W.J., Seong, H.J., Rhee, C., Ekpheghere, K.I., Kim, I.S., Kim, H.G., Koh, S.C. 2019 b. Metagenomic analysis of relationships between the denitrification process and carbon metabolism in a bioaugmented full-scale tannery wastewater treatment plant. World Journal of Microbiology \& Biotechnology, 35(10), 12. https://doi.org/10.1007/s11274-019-2716-8

12. Garrido-Sanz, D., Manzano, J., Martin, M., Redondo-Nieto, M., Rivilla, R. 2018. Metagenomic Analysis of a Biphenyl-Degrading Soil Bacterial Consortium Reveals the Metabolic Roles of Specific Populations. Front Microbiol, 9, 232. https://doi.org/10.3389/fmicb.2018.00232

13. Gonzalez-Martinez, A., Sihvonen, M., Munoz-Palazon, B., Rodriguez-Sanchez, A., Mikola, A., Vahala, R. 2018. Microbial ecology of full-scale wastewater treatment systems in the Polar Arctic Circle: Archaea, Bacteria and Fungi. Sci Rep, 8(1), 2208. https://doi.org/10.1038/s41598-018-20633-5

14. Goudarzi, M., Weber, W.M., Mak, T.D., Chung, J., Doyle-Eisele, M., Melo, D.R., Strawn, S.J., Brenner, D.J., Guilmette, R.A., Fornace, A.J., Jr. 2015. A Comprehensive Metabolomic Investigation in Urine of Mice Exposed to Strontium-90. Radiat Res, 183(6), 665-74. https://doi.org/10.1667/RR14011.1 
15. Greses, S., Zamorano-Lopez, N., Borras, L., Ferrer, J., Seco, A., Aguado, D. 2018. Effect of long residence time and high temperature over anaerobic biodegradation of Scenedesmus microalgae grown in wastewater. $J$ Environ Manage, 218, 425-434. https://doi.org/10.1016/j.jenvman.2018.04.086

16. He, G., Wu, C., Huang, J., Zhou, R. 2016. Acid tolerance response of Tetragenococcus halophilus: A combined physiological and proteomic analysis. Process Biochemistry, 51(2), 213-219. https://doi.org/10.1016/j.procbio.2015.11.035

17. Huang, F., Mei, X.-j., Wang, Z.-w., Wu, Z.-C. 2014. Diversity of Operation Performance and Microbial Community Structures in MBRs and CAS Processes at Low Temperature. Huanjing Kexue, 35(3), 1002-1008.

18. Hug, L.A., Castelle, C.J., Wrighton, K.C., Thomas, B.C., Sharon, I., Frischkorn, K.R., Williams, K.H., Tringe, S.G., Banfield, J.F. 2013. Community genomic analyses constrain the distribution of metabolic traits across the Chloroflexi phylum and indicate roles in sediment carbon cycling. Microbiome, 1, 17. https://doi.org/10.1186/2049-2618-1-22

19. Jin, L.Y., Zhang, G.M., Tian, H.F. 2014. Current state of sewage treatment in China. Water Research, 66, 85-98. https://doi.org/10.1016/j.watres.2014.08.014

20. Johnston, J., Behrens, S. 2020. Seasonal Dynamics of the Activated Sludge Microbiome in Sequencing Batch Reactors, Assessed Using 16S rRNA Transcript Amplicon Sequencing. Appl Environ Microbiol, 86(19). https://doi.org/10.1128/AEM.00597-20

21. Johnston, J., LaPara, T., Behrens, S. 2019. Composition and Dynamics of the Activated Sludge Microbiome during Seasonal Nitrification Failure. Sci Rep, 9(1), 4565. https://doi.org/10.1038/s41598-019-40872-4

22. Ju, F., Guo, F., Ye, L., Xia, Y., Zhang, T. 2014. Metagenomic analysis on seasonal microbial variations of activated sludge from a full-scale wastewater treatment plant over 4 years. Environ Microbiol Rep, 6(1), 80-9. https://doi.org/10.1111/1758-2229.12110

23. Kampschreur, M.J., Temmink, H., Kleerebezem, R., Jetten, M.S., van Loosdrecht, M.C. 2009. Nitrous oxide emission during wastewater treatment. Water Res, 43(17), 4093-103. https://doi.org/10.1016/j.watres.2009.03.001

24. Kodner, R.B., Summons, R.E., Pearson, A., King, N., Knoll, A.H. 2008. Sterols in a unicellular relative of the metazoans. Proc Natl Acad Sci U S A, 105(29), 9897-902. https://doi.org/10.1073/pnas.0803975105

25. Kraemer, P., Gerlach, G., Brinkhoff, T. 2020. Diversity and flexibility of the bacterial communities on Cancer pagurus at different temperatures. Journal of Sea Research, 156.

https://doi.org/10.1016/j.seares.2019.101827

26. Kuang, S., Fan, X., Peng, R. 2018. Quantitative proteomic analysis ofRhodococcus ruberresponsive to organic solvents. Biotechnology \& Biotechnological Equipment, 32(6), 1418-1430. https://doi.org/10.1080/13102818.2018.1533432

27. Laffont, C., Arnoux, P. 2020. The ancient roots of nicotianamine: diversity, role, regulation and evolution of nicotianamine-like metallophores. Metallomics, 12(10), 1480-1493. https://doi.org/10.1039/d0mt00150c

28. Latif, M.A., Mehta, C.M., Batstone, D.J. 2015. Low pH anaerobic digestion of waste activated sludge for enhanced phosphorous release. Water Research, 81, 288-293. https://doi.org/10.1016/j.watres.2015.05.062

29. Liu, L., Ji, M., Wang, F., Tian, Z., Yan, Z., Wang, S. 2021. N-acyl-I-homoserine lactones release and microbial community changes in response to operation temperature in an anammox biofilm reactor. Chemosphere, 262, 127602. https://doi.org/10.1016/j.chemosphere.2020.127602 
30. Liu, T., Liu, S., Zheng, M., Chen, Q., Ni, J. 2016. Performance Assessment of Full-Scale Wastewater Treatment Plants Based on Seasonal Variability of Microbial Communities via High-Throughput Sequencing. PLoS One, 11(4), e0152998. https://doi.org/10.1371/journal.pone.0152998

31. Ohata, T., Kanazawa, K., Mihashi, S., Kishi-Nishizawa, N., Fushiya, S., Nozoe, S., Chino, M., Mori, S. 1993. Biosynthetic pathway of phytosiderophores in iron-deficient graminaceous plants. Soil Science and Plant Nutrition, 39(4), 745-749. https://doi.org/10.1080/00380768.1993.10419193

32. Park, J.H., Lee, S.Y. 2010. Metabolic pathways and fermentative production of L-aspartate family amino acids. Biotechnol J, 5(6), 560-77. https://doi.org/10.1002/biot.201000032

33. Pramanik, B., Suja, F., Zain, S., Elshafie, A. 2012. Biological aerated filters (BAFs) for carbon and nitrogen removal: A review. Journal of Engineering Science and Technology, 7, 534-553.

34. Ross, B.N., Wigginton, S.K., Cox, A.H., Loomis, G.W., Amador, J.A. 2020. Influence of Season, Occupancy Pattern, and Technology on Structure and Composition of Nitrifying and Denitrifying Bacterial Communities in Advanced Nitrogen-Removal Onsite Wastewater Treatment Systems. Water, 12(9). https://doi.org/10.3390/w12092413

35. Roume, H., Heintz-Buschart, A., Muller, E.E.L., May, P., Satagopam, V.P., Laczny, C.C., Narayanasamy, S., Lebrun, L.A., Hoopmann, M.R., Schupp, J.M., Gillece, J.D., Hicks, N.D., Engelthaler, D.M., Sauter, T., Keim, P.S., Moritz, R.L., Wilmes, P. 2015. Comparative integrated omics: identification of key functionalities in microbial community-wide metabolic networks. NPJ Biofilms Microbiomes, 1, 15007.

https://doi.org/10.1038/npjbiofilms.2015.7

36. Samuilov, S., Brilhaus, D., Rademacher, N., Flachbart, S., Arab, L., Alfarraj, S., Kuhnert, F., Kopriva, S., Weber, A.P.M., Mettler-Altmann, T., Rennenberg, H. 2018. The Photorespiratory BOU Gene Mutation Alters Sulfur Assimilation and Its Crosstalk With Carbon and Nitrogen Metabolism in Arabidopsis thaliana. Front Plant Sci, 9, 1709. https://doi.org/10.3389/fpls.2018.01709

37. Shi, R., Xu, S., Qi, Z., Huang, H., Liang, Q. 2019. Seasonal patterns and environmental drivers of nirS- and nirKencoding denitrifiers in sediments of Daya Bay, China. Oceanologia, 61(3), 308-320.

https://doi.org/10.1016/j.oceano.2019.01.002

38. Takahashi, M., Yamaguchi, H., Nakanishi, H., Shioiri, T., Nishizawa, N.-K., Mori, S. 1999. Cloning Two Genes for Nicotianamine Aminotransferase, a Critical Enzyme in Iron Acquisition (Strategy II) in Graminaceous Plants. Plant Physiology, 121(3), 947-956. https://doi.org/10.1104/pp.121.3.947

39. Tao, C., Peng, T., Feng, C., Chen, N., Hu, Q., Hao, C. 2016. The feasibility of an up-flow partially aerated biological filter (U-PABF) for nitrogen and COD removal from domestic wastewater. Bioresour Technol, 218, 307-17. https://doi.org/10.1016/j.biortech.2016.06.098

40. Tian, L., Wang, L. 2019. Debugging Operation of Pre-denitrification Biological Aerated Filter. Technology of Water Treatment, 45(12), 129-132.

41. Tian, L., Wang, L. 2020. A meta-analysis of microbial community structures and associated metabolic potential of municipal wastewater treatment plants in global scope. Environmental Pollution, 263, 11. https://doi.org/10.1016/j.envpol.2020.114598

42. Tian, L., Wang, L. 2021. Multi-omics analysis reveals structure and function of biofilm microbial communities in a pre-denitrification biofilter. Science of the Total Environment, 757, 12.

https://doi.org/10.1016/j.scitotenv.2020.143908

Page 15/23 
43. Uribe-Lorio, L., Brenes-Guillen, L., Hernandez-Ascencio, W., Mora-Amador, R., Gonzalez, G., Ramirez-Umana, C.J., Diez, B., Pedros-Alio, C. 2019. The influence of temperature and pH on bacterial community composition of microbial mats in hot springs from Costa Rica. Microbiologyopen, 8(10), e893.

https://doi.org/10.1002/mbo3.893

44. Wang, J., Chu, L. 2016. Biological nitrate removal from water and wastewater by solid-phase denitrification process. Biotechnol Adv, 34(6), 1103-1112. https://doi.org/10.1016/j.biotechadv.2016.07.001

45. Wang, J., Liu, Q., Hu, H., Wu, B., Zhang, X.X., Ren, H. 2019. Insight into mature biofilm quorum sensing in fullscale wastewater treatment plants. Chemosphere, 234, 310-317.

https://doi.org/10.1016/j.chemosphere.2019.06.007

46. Wang, P., Yu, Z., Qi, R., Zhang, H. 2016. Detailed comparison of bacterial communities during seasonal sludge bulking in a municipal wastewater treatment plant. Water Res, 105, 157-166.

https://doi.org/10.1016/j.watres.2016.08.050

47. Wang, X.H., Hu, M., Xia, Y., Wen, X.H., Ding, K. 2012. Pyrosequencing Analysis of Bacterial Diversity in 14 Wastewater Treatment Systems in China. Applied and Environmental Microbiology, 78(19), 7042-7047. https://doi.org/10.1128/aem.01617-12

48. Ward, L.M., Hemp, J., Shih, P.M., McGlynn, S.E., Fischer, W.W. 2018. Evolution of Phototrophy in the Chloroflexi Phylum Driven by Horizontal Gene Transfer. Front Microbiol, 9, 260. https://doi.org/10.3389/fmicb.2018.00260

49. Werner, J.J., Knights, D., Garcia, M.L., Scalfone, N.B., Smith, S., Yarasheski, K., Cummings, T.A., Beers, A.R., Knight, R., Angenent, L.T. 2011. Bacterial community structures are unique and resilient in full-scale bioenergy systems. Proc Natl Acad Sci U S A, 108(10), 4158-63. https://doi.org/10.1073/pnas.1015676108

50. Xiao, Y., Wang, Y. 2016. Global discovery of protein kinases and other nucleotide-binding proteins by mass spectrometry. Mass Spectrom Rev, 35(5), 601-19. https://doi.org/10.1002/mas.21447

51. Xu, H., Ma, R., Zhu, Y., Du, M., Zhang, H., Jiao, Z. 2020. A systematic study of the antimicrobial mechanisms of cold atmospheric-pressure plasma for water disinfection. Sci Total Environ, 703, 134965.

https://doi.org/10.1016/j.scitotenv.2019.134965

52. Zhang, H., Feng, J., Chen, S., Zhao, Z., Li, B., Wang, Y., Jia, J., Li, S., Wang, Y., Yan, M., Lu, K., Hao, H. 2019. Geographical Patterns of nirS Gene Abundance and nirS-Type Denitrifying Bacterial Community Associated with Activated Sludge from Different Wastewater Treatment Plants. Microb Ecol, 77(2), 304-316. https://doi.org/10.1007/s00248-018-1236-7

53. Zhang, Q.H., Yang, W.N., Ngo, H.H., Guo, W.S., Jin, P.K., Dzakpasu, M., Yang, S.J., Wang, Q., Wang, X.C., Ao, D. 2016. Current status of urban wastewater treatment plants in China. Environ Int, 92-93, 11-22. https://doi.org/10.1016/j.envint.2016.03.024

54. Zhang, S., Fan, F., Meng, F. 2020. Seasonality and Community Separation of Fungi in a Municipal Wastewater Treatment Plant. App/ Environ Microbiol, 86(18). https://doi.org/10.1128/AEM.00991-20

55. Zhao, L., Zhang, H., White, J.C., Chen, X., Li, H., Qu, X., Ji, R. 2019. Metabolomics reveals that engineered nanomaterial exposure in soil alters both soil rhizosphere metabolite profiles and maize metabolic pathways. Environmental Science: Nano, 6(6), 1716-1727. https://doi.org/10.1039/c9en00137a

56. Zhong, Q., Wang, B., Wang, J., Liu, Y., Fang, X., Liao, Z. 2019. Global Proteomic Analysis of the Resuscitation State of Vibrio parahaemolyticus Compared With the Normal and Viable but Non-culturable State. Front Microbiol, 10, 1045. https://doi.org/10.3389/fmicb.2019.01045 
57. Zhou, H., Xu, G. 2020. Biofilm characteristics, microbial community structure and function of an up-flow anaerobic filter-biological aerated filter (UAF-BAF) driven by COD/N ratio. Sci Total Environ, 708, 134422. https://doi.org/10.1016/j.scitotenv.2019.134422

58. Zhou, H., Xu, G. 2019. Integrated effects of temperature and COD/N on an up-flow anaerobic filter-biological aerated filter: Performance, biofilm characteristics and microbial community. Bioresour Technol, 293, 122004. https://doi.org/10.1016/j.biortech.2019.122004

59. Zhou, Y., Li, R., Guo, B., Zhang, L., Zou, X., Xia, S., Liu, Y. 2020. Greywater treatment using an oxygen-based membrane biofilm reactor: Formation of dynamic multifunctional biofilm for organics and nitrogen removal. Chemical Engineering Journal, 386. https://doi.org/10.1016/j.cej.2019.123989

\section{Figures}



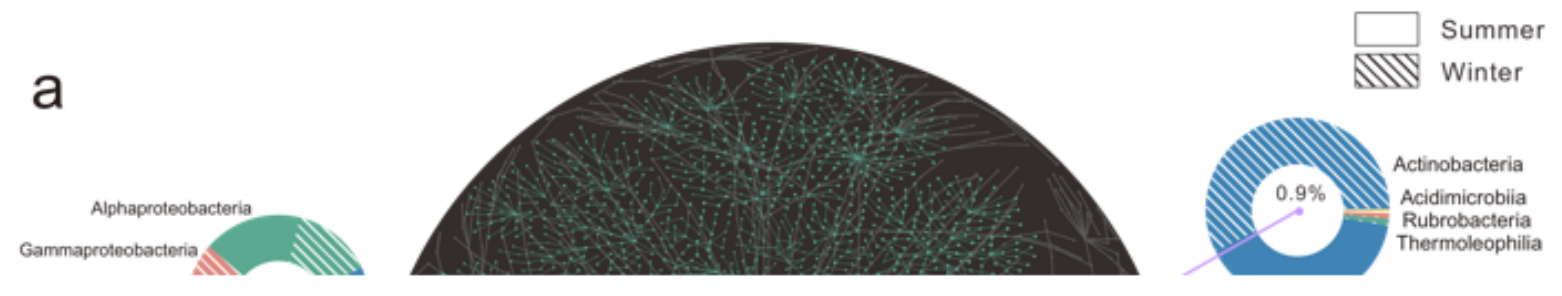

\section{Figure 1}

Composition of biofilm microbial communities. (a) Comparison of the composition of microbial communities at the phylum level in winter and summer samples based on metagenomic data. Comparison of the top 10 abundant bacterial classes identified by metagenomic and metaproteomic in (b) summer and (c) winter samples. 

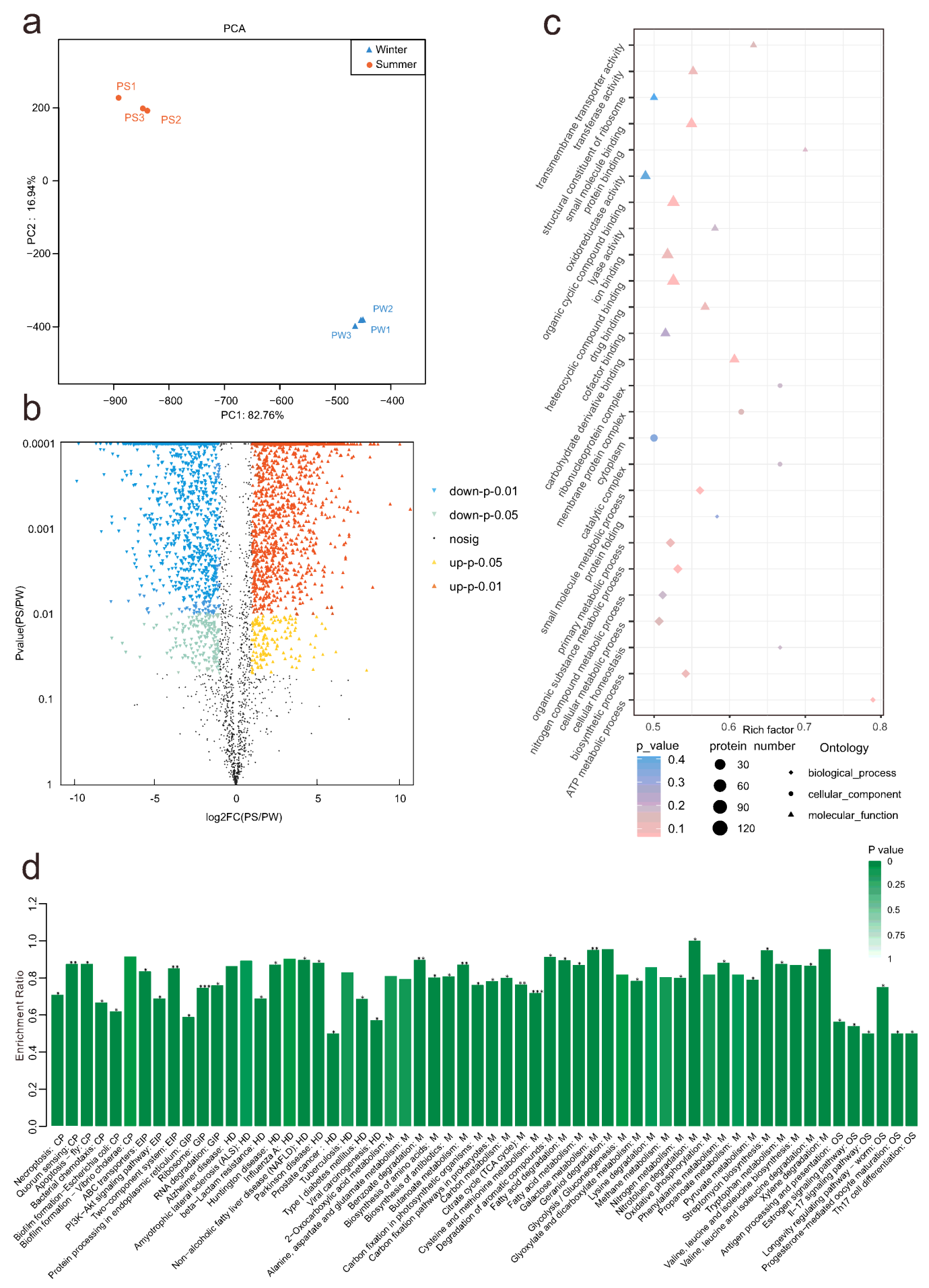

Figure 2

(a) Score plot of Principal Component Analysis of identified proteins. (b) Volcano plot of differentially expressed proteins between summer and winter groups. (c) GO enrichment analyses of all differentially expressed proteins. Each bubble represented one $\mathrm{GO}$ term. Size of bubbles represents the number of proteins, and color of the bubbles was related to the p-value. (d) KEGG pathway enrichment of DEPs. Each bar represented one KEGG pathway. Pathway was marked ${ }^{* \star} p<0.001,{ }^{\star \star} p<0.05$ and ${ }^{\star} p<0.01$. Color of the bars was related to the $p$-value. Decreasing of the $\mathrm{p}$-value was corresponded to the color from light to dark. 

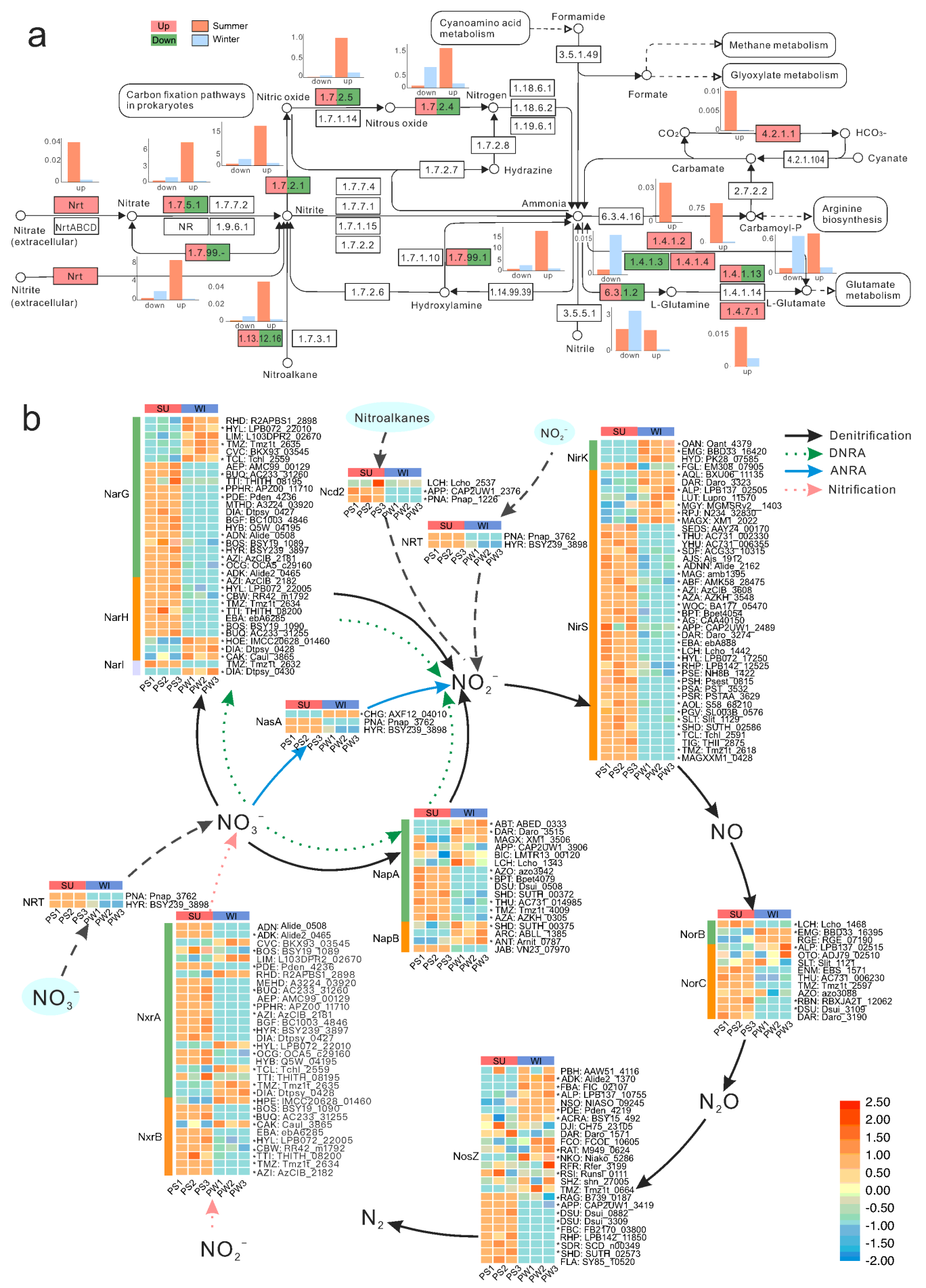

\section{Figure 3}

Nitrogen metabolism involved differentially expressed proteins expression. (a) Nitrogen metabolism pathway map. Red and green boxes indicate up-regulated and down-regulated enzymes, respectively. The bar chart shows the comparison of enzyme abundance between the two groups. (b) Protein expression profiles of genes encoding specific enzymes for nitrogen metabolism. The protein expression profiles with significant differences between summer and winter samples are marked with asterisks. 

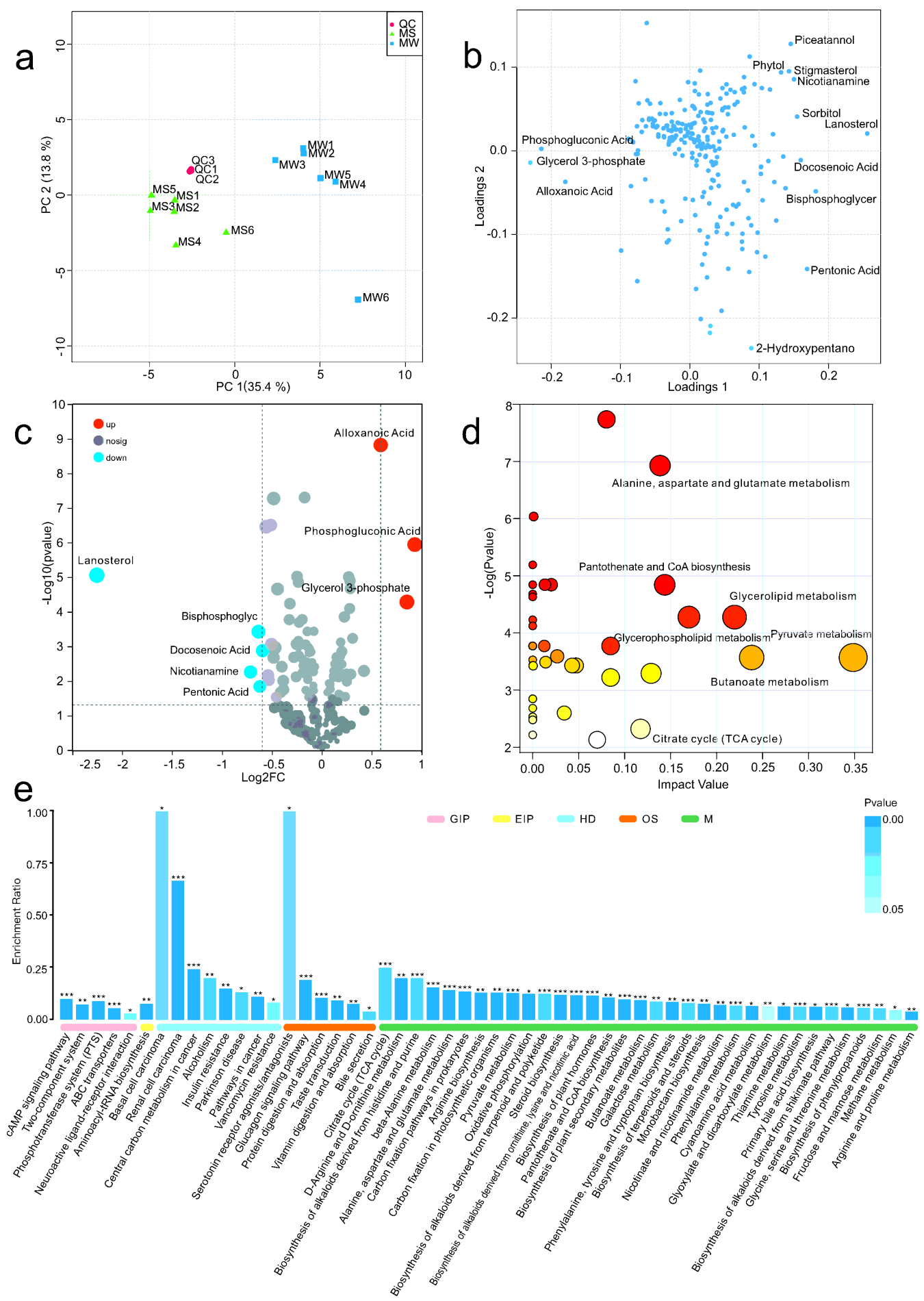

\section{Figure 4}

Metabolomic profiles of biofilm samples from summer and winter groups. Principal Component Analysis (a) score plot and (b) loading plot. QC, quality control group; MS, Summer group; MW, winter group. (c) Volcano plots of significantly regulated metabolites. The metabolites significantly down-regulated in expression $(\mathrm{FC}<0.67)$ are labeled in blue and those significantly up-regulated in expression (FC > 1.5) are labeled in orange. (d) Pathway topology analysis based on the identified differential metabolites. Larger sizes and darker colors represent greater pathway enrichment and higher pathway impact values, respectively. 


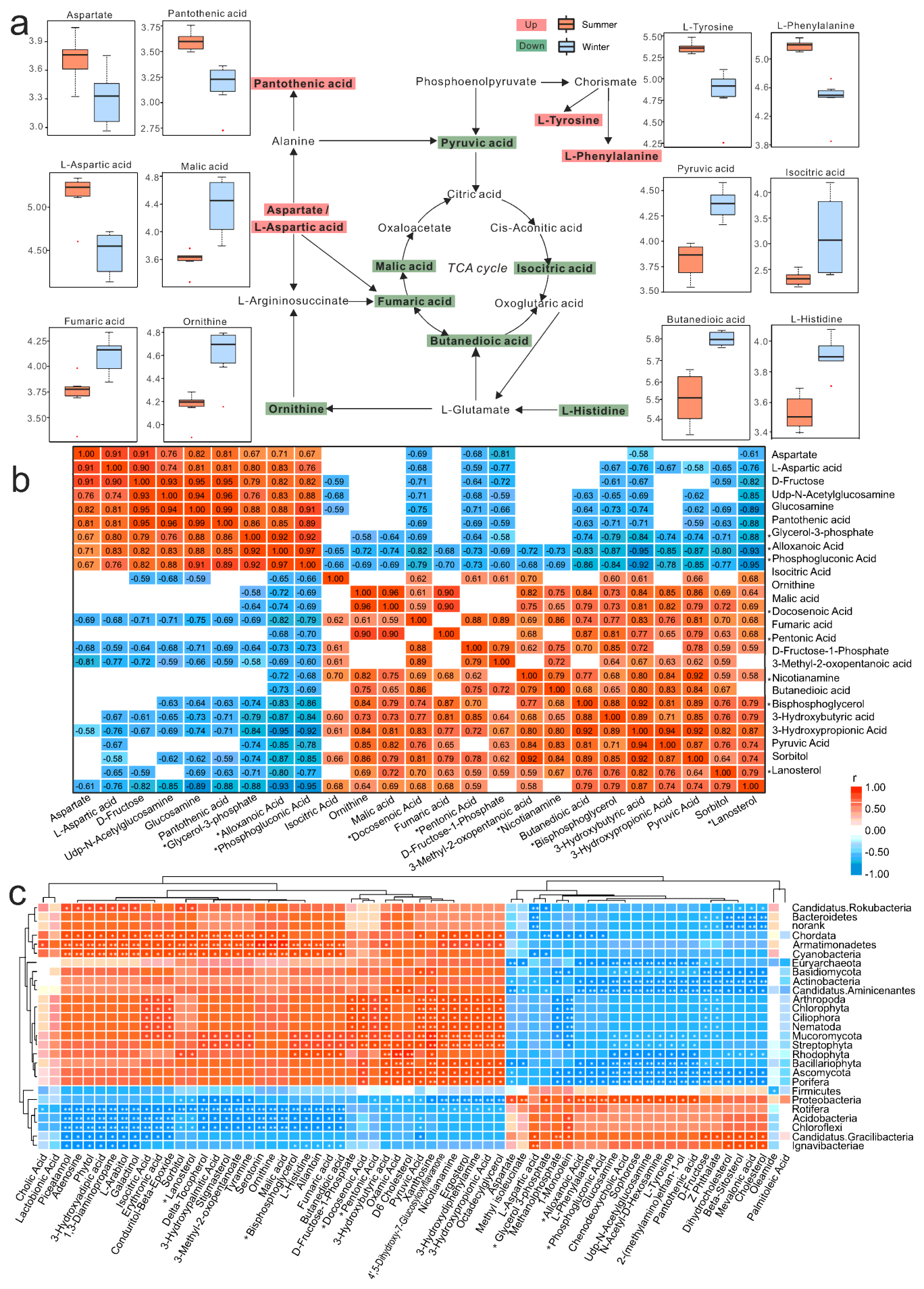

Figure 5

(a) significantly perturbed amino acids and organic acids in response to season variations. Red text, Up-regulated differential metabolites compared to the winter group; green text, down-regulated differential metabolites compared to the winter group. The bar chart shows the comparison of metabolites concentration between the two groups. (b) Correlation heatmap of potential biomarkers and perturbed metabolites. Threshold values of $|r|>0.4$ and $P<0.05$. Potential biomarkers are marked * in front of the name. (c) Correlation heatmap of microbial communities and differential metabolites. Potential biomarkers are marked * in front of the name. Orange represents a positive correlation, while blue represents a negative correlation. significant correlation: $\mathrm{P}<0.01$ : **; $P<0.05: *$. 


\section{Supplementary Files}

This is a list of supplementary files associated with this preprint. Click to download.

- supplementarymaterials.docx 